\title{
La dynamique contemporaine de sécurité et le renouveau de la défense civile américaine sous l'administration Clinton
}

The contemporary dynamics of security and the renewal of US civil defense under Clinton administration

Olivier Palluault

\section{(2) OpenEdition Journals}

\section{Édition électronique}

URL : http://journals.openedition.org/conflits/18256

DOI : $10.4000 /$ conflits. 18256

ISSN : $1777-5345$

\section{Éditeur :}

CCLS - Centre d'études sur les conflits lilberté et sécurité, L'Harmattan

\section{Édition imprimée}

Date de publication : 31 décembre 2011

Pagination : 103-129

ISBN : 978-2-296-96617-8

ISSN : $1157-996 X$

\section{Référence électronique}

Olivier Palluault, « La dynamique contemporaine de sécurité et le renouveau de la défense civile américaine sous l'administration Clinton », Cultures \& Conflits [En ligne], 84 | Hiver 2011, mis en ligne le 15 mars 2013, consulté le 30 mars 2021. URL : http://journals.openedition.org/conflits/18256 ; DOI : https://doi.org/10.4000/conflits.18256 


\section{La dynamique contemporaine de sécurité et le renouveau de la défense civile américaine sous I'administration Clinton}

\section{Olivier PALLUAULT}

Olivier Palluault est docteur en science politique, diplômé de l'Institut d'Études Politiques de Paris et diplômé d'études approfondies en sociologie politique à l'université Paris $X$. Sa thèse, sous la direction du professeur Pascal Vennesson, a été soutenue en 2009 et a reçu le prix en science politique de l'université Paris II Panthéon Assas. Olivier Palluault a bénéficié de l'allocation de recherche du CNRS et de la Délégation Générale de l'Armement. Il a été visiting fellow à l'université George Washington au sein de l'Elliott School for International Affairs (2004-2005). Ses travaux portent sur les relations internationales, les transformations de la sécurité et les politiques publiques.

T a transformation contemporaine de la sécurité renvoie, entre autres, à la ـrésurgence de missions de défense civile et de protection du territoire, à la dissipation des limites entre le policier et le militaire, à une déterritorialisation des pratiques relevant de la sécurité nationale, à un maillage territorial en deçà et au-delà des frontières nationales par des technologies de surveillance et d'identification ${ }^{1}$. Elle est désignée depuis le 11 septembre 2001 comme intimement liée à la menace du terrorisme, aux dispositifs et aux pratiques élaborés et mis en place pour y faire face ${ }^{2}$. Aux États-Unis, la nouvelle stratégie de la homeland security, établie en 2002 et la création l'année suivante d'un nouveau ministère mis en place spécifiquement pour l'appliquer, contribuent à cette impression qu'une nouvelle donne sécuritaire s'est instaurée avec l'effondrement des tours jumelles de New York, du moins sur le plan de l'organisation de la sécurité intérieure ${ }^{3}$. Toute une littérature scientifique s'est concen-

1. Sur ces enjeux appliqués à la sécurité intérieure américaine, Kettl D.F., System under Stress : Homeland Security and American Politics, Washington, CQ Press, 2004 ; Brzezinski M., Fortress America: On the Front Lines of Homeland Security: An Inside Look at the Coming Surveillance State, New York, Bantam Books, 2004.

2. Lansford T., Pauly R.J, Covarrubias J., To Protect and Defend: US Homeland Security Policy, Aldershot, Ashgate, 2006.

3. The National Strategy for Homeland Security, Washington, The White House, Office of Homeland Security, juillet 2002. La traduction française de Homeland Security par « sécurité 
trée sur les effets de cette transformation et les formes qu'elle prend en matière de normes, de pratiques, de discours ou de dispositifs technologiques ${ }^{4}$. Elle semble avoir délaissé toutefois la question de la production de cette transformation sécuritaire, c'est-à-dire non seulement les conditions qui l'ont rendue possible, mais également comment elle s'est concrètement constituée.

Cet article entend examiner les ressorts de cette transformation, interroger la nature de son apparition et son développement. Il s'appuie sur l'analyse des programmes de défense civile élaborés et mis en place après la Guerre froide sous l'administration Clinton, et en premier lieu la politique de protection des infrastructures critiques ${ }^{5}$. Ce programme, établi au milieu des années quatre-vingt-dix, visant principalement à protéger la société américaine contre les attaques informatiques stratégiques et le « cyberterrorisme », a été érigé en quelques années au rang de priorité de la sécurité nationale américaine, avant de devenir l'un des piliers de la homeland security après les attentats du 11 septembre ${ }^{6}$.

Pour rendre compte des fondements de la transformation de la sécurité, cet article pose comme hypothèse principale que les années qui ont immédiatement suivi la Guerre froide ont vu se structurer et se renforcer des évolutions spécifiques au sein du champ de la sécurité, des évolutions qui ont concouru à la construction d'un nouveau type de sécurité : située au-delà du militaire et du policier, promue par de nouveaux experts surfant sur des dis-

intérieure » ou «protection du territoire » est impropre car elle dissimule les dimensions transnationales et identitaires que le concept anglo-saxon enferme. Aussi conservons-nous le terme anglophone dans cet article. Voir sur ce point Ceyhan A., « Sécurité, frontières et surveillance aux États-Unis après le 11 septembre 2001 », Cultures E Conflits, 53, 2004, pp.113145.

4. Pour des approches critiques et sociologiques de cette transformation, Muller B., Security, Risk and the Biometric State: Governing Borders and Bodies, Londres et New York, Routledge, 2010 ; Bigo D., Tsoukala A. (eds.), Terror, Insecurity and Liberty: Illiberal Practices of Liberal Regimes after 9/11, Londres et New York, Routledge, 2008 ; RollingsMagnusson S. (ed.), Anti-Terrorism: Security and Insecurity after 9/11, Halifax, Fernwood Publishing, 2009.

5. Palluault O., Stratégie de la précaution: la construction de la protection des infrastructures critiques aux États-Unis de 1993 à 2003, thèse de doctorat de l'université Paris II PanthéonAssas, Paris, 2009. Voir également Dunn Cavelty M., Kristensen K.S., Securing "the Homeland": Critical Infrastructure, Risk and (In)security, London - New York, Routledge, 2008. Le second programme de défense civile établi dans les années quatre-vingt-dix comprend les mesures contre des attentats commis sur le territoire américain à l'aide d'une arme de destruction massive.

6. Selon la définition du président Clinton en 1996, les infrastructures critiques sont celles dont « la destruction ou la paralysie aurait un effet incapacitant et funeste pour la défense nationale ou la sécurité économique du pays. » William J. Clinton, "Executive Order 13010: Critical Infrastructure Protection”, Federal Register, vol.61, n¹38, 17 juillet 1996, p. 37345-37450. La stratégie de sécurité nationale érige la protection des infrastructures critiques dès 1998 au rang d'intérêt vital de la nation américaine. Voir William J. Clinton, The National Security Strategy for A New Century, Washington DC: The White House, 1998, p. 5. En réalité, cette politique s'assimile initialement à un programme de sécurité contre les attaques informatiques. Sur ce point, voir Palluault O., Stratégie de la précaution : la construction de la protection des infrastructures critiques aux États-Unis de 1993 à 2003, op.cit., pp.109-139. 
cours de rupture avec la période de la Guerre froide, cette sécurité contemporaine s'incarne dans les nouveaux systèmes technologiques de surveillance et d'identification, et les divers dispositifs de résilience et de réduction des vulnérabilités. Pour étayer cette hypothèse, cet article présente une méthodologie originale, autour du concept de «dynamique de sécurité », qui vise à comprendre et analyser les phénomènes de changement appliqués aux enjeux de sécurité. Cette méthodologie prête une attention particulière aux formes de discontinuités qui s’opèrent au sein du champ de la sécurité à travers divers processus sociaux. Ces processus ont trait à la manière de penser et de dire la sécurité (processus ontologique), aux pratiques de sécurité (processus stratégique), à l'organisation bureaucratique de l'État relative à ses missions de sécurité (processus bureaucratique), aux itinéraires et aux stratégies des acteurs de la sécurité (processus sociologique), à l'appréhension et à l'utilisation des technologies de sécurité (processus technologique). Les périodes de transformation, qui s'opèrent sous la forme de dynamiques, apparaissent à l'occasion d'une convergence entre ces cinq processus. Nous soutenons ainsi que des évolutions convergentes se sont produites au sein de ces cinq processus dans le contexte de l'après Guerre froide et ont abouti à former aux ÉtatsUnis une dynamique de sécurité qui s'est incarnée dans le renouveau de la défense civile américaine à partir du milieu des années quatre-vingt-dix et a structuré les politiques sécuritaires de l'après-11 septembre.

Cet article est divisé en trois parties. La première présentera rapidement les soubassements théoriques du concept de dynamique de sécurité et les enjeux d'analyse qu'il enferme, notamment dans le prolongement du concept de sécurisation avancé par Ole Waever. La seconde présentera l'évolution des différents processus sociaux appliqués au champ de la sécurité dans le contexte de l'après Guerre froide. Enfin, la troisième partie nous permettra de cerner la dynamique contemporaine de la sécurité, et de présenter une interprétation des réformes mises en place à la suite des attentats du 11 septembre.

\section{Qu'est ce qu'une dynamique de sécurité ?}

Le concept de «dynamique de sécurité » s’inscrit dans le prolongement des travaux menés depuis une vingtaine d'années par les constructivistes à l'encontre des positions rationalistes des réalistes, qui présentent la transformation sécuritaire comme le reflet des pratiques d'insécurité et de production du danger ${ }^{7}$. Une dynamique de sécurité est une matrice qui travaille et modifie le champ de la sécurité ${ }^{8}$. Elle est elle-même créée à partir des (épi-) phéno-

7. Parmi les ouvrages de référence, voir Krause K., Williams M., Critical Security Studies: Concepts and Cases, Minneapolis, University of Minnesota Press, 1997 ; Weldes J., Laffey M., Gusterson H. (eds.), Cultures of Insecurity: States, Communities, and the Production of Danger, Minneapolis, University of Minnesota Press, 1999. Pour un résumé des débats contemporains, voir Buzan B., Hansen L., The Evolution of International Security Studies, Cambridge, Cambridge University Press, 2009.

8. Sur la notion de champ de la sécurité, voir Bigo D., Terrorisme, guerre, sécurité intérieure et 
mènes qui apparaissent au sein de ce champ mais qui, par leurs interactions, produisent un cadre structurel qui va donner une cohérence aux changements qui, sans ce cadre, apparaissent épars et désordonnés.

\section{Une sociologie politique de la sécurisation}

Le concept de dynamique sécuritaire se veut initialement une contribution à la théorie de la sécurisation avancée par Ole Waever au milieu des années quatre-vingt-dix, qui vise à comprendre comment une question d'ordre technique, social, politique, culturel ou moral devient un enjeu sécuritaire jusqu'à faire l'objet d'un programme politique spécifique, élaboré à partir d'une vision militaire ou policière 9 . Pour Waever, la sécurité est créée par des acteurs dominants au travers des pratiques discursives qui construisent - en même temps qu'elles décrivent - une situation particulière. C'est le discours sécuritaire, le speech act, qui constitue la sécurité, en imposant à des objets référents des caractéristiques dont elles étaient auparavant dépourvues : en labellisant des objets sous l'appellation "sécurité », des décideurs publics modifient la perception de ces objets et appellent à un traitement politique fait d'exception et d'urgence ${ }^{10}$. La conceptualisation d'une situation et sa représentation à travers le discours sécuritaire auprès d'une audience constituent alors la sécurisation.

Aussi fondatrice et influente qu'elle fut, la théorie de Waever est problématique en ce qu'elle ne permet pas de comprendre comment une pratique discursive parvient réellement à transformer une question en problème sécuritaire ${ }^{11}$. Les premiers écrits de Waever sous-entendent en effet que toute question peut avec succès être sécurisée, indépendamment de son statut, à partir du moment où elle est reliée à une menace présentée comme existentielle. Par ailleurs, Waever mentionne de manière assez floue les représentants de l'État comme les acteurs disposant de l'autorité suffisante pour asseoir le processus de sécurisation auprès d'une audience, sans toutefois analyser plus avant qui sont ces acteurs, de quelles positions ils parlent, de quelle autorité ils sont investis, comment et à partir de quoi ils ont formé leurs propres actes de parole ${ }^{12}$. Cette question est pourtant centrale car elle détermine « qui peut exprimer la sécurité légitimement et avec succès 13 ».

sécurité extérieure. Mémoire d'habilitation à diriger des recherches, Institut d'Études Politiques de Paris, décembre 2002. Le terme de matrice renvoie à la capacité génératrice de la dynamique : elle « produit » des politiques sécuritaires.

9. Waever O., "Securitization and Desecuritization", in Lipschutz R., On security, New York, Columbia University Press, 1995, pp. 46-86. Voir également Waever O., Buzan B., de Wilde J., Security: A New Framework for Analysis, Boulder, Colorado: Rienner, 1998.

10. Waever O., "Securitization and Desecuritization", op.cit., p. 55.

11. Sur les critiques contre Waever, voir notamment McSweeney B., Security, Identity and Interests: a Sociology of International Relations, Cambridge, Cambridge University Press, 1999 ; Ceyhan A., "Analyser la sécurité : Dillon, Waever, Williams et les autres ", Cultures E Conflits, vol.31-32, 1997, pp. 39-62 ; Huysmans J., « Dire et écrire la sécurité : le dilemme normatif des études de sécurité », Cultures E Conflits, vol.31-32, pp. 177-202. 
Le concept de dynamique de sécurité reprend les apports fondamentaux des constructivistes critiques à l'encontre du concept de Waever. S'il reconnait aux actes de langage une dimension cruciale dans le processus de sécurisation, il s'attache à mieux préciser les conditions dans lesquelles ils sont produits ${ }^{14}$. Ces actes ne prennent sens que dans le cadre d'une dynamique sociale complexe qui conditionne sa possibilité (c'est-à-dire le discours du locuteur, mais aussi la présence, l'écoute et la composition de l'auditoire), qui détermine la relation entre le locuteur et son auditoire et la réception du discours sous forme de rejet, d'approbation, de scepticisme, ou d'indifférence, et qui conditionne in fine le critère d'urgence de l'objet sur l'échelle des impératifs sécuritaires ${ }^{15}$. Surtout, ces actes ne sauraient suffire pour produire de la sécurité, et doivent s'appréhender aux côtés d'autres pratiques non discursives, telles que le recours aux images ou le travail de routinisation des professionnels de la sécurité ${ }^{16}$. Concernant le cas qui nous intéresse, nous verrons plus loin comment la sécurisation des thèmes portant sur la sécurité informatique stratégique s'est opérée à partir de multiples lieux et à travers des procédés qui relèvent des discours politiques, des discours savants, des univers métaphoriques et symboliques largement diffusés dans les médias et la littérature profane, ainsi que des pratiques non-discursives de gestion de la menace, au premier rang desquelles la sélection et l'interprétation des données sur les « attaques » informatiques ou la construction des jeux de guerre ${ }^{17}$. Tandis qu'Ole Waever laisse croire à la seule capacité d'instrumentalisation des acteurs dominants dans la construction de la sécurité, le renouveau de la défense civile américaine s'est plutôt opéré depuis les marges, par le fait d'acteurs à qui l'on déniait auparavant toute autorité à parler de la sécurité. De surcroît, excluant les facteurs qui agissent à la fois en amont et en aval de l'acte de parole, Weaver réduit la sécurisation à une temporalité courte - à un instant - en privilégiant le moment de dramatisation que constitue la prise de décision ${ }^{18}$. Une fois

12. Huysmans J., «Dire et écrire la sécurité : le dilemme normatif des études de sécurité », op.cit., p. 196.

13. Ibid, p. 194

14. La critique principale à l'approche de Waever, même amendée dans l'ouvrage de 1998 co-écrit avec Barry Buzan et Jaap de Wilde, porte sur sa faiblesse sociologique. Sur ce débat, voir Balzacq T., "Securitization Theory: Origins, Core Assumptions and Variants", in Balzacq T. (ed.), Securitization Theory: How Security Problems Emerge and Dissolve, New York, Routledge, 2011, pp. 1-30. Voir aussi Huysmans J., "Revisiting Copenhagen, or, About the Creative Development of a Security Studies Agenda in Europe", European Journal of International Relations, vol.4, n4, 1997, pp. 488-506.

15. Balzacq T., "The Three Faces of Securitization: Political Agency, Audience and Context", European Journal of International Relations, vol.11, n², 2005, pp. 171-201.

16. Sur le recours aux images comme procédé de sécurisation, voir Williams M., "Words, Images, Enemies: Securitization and International Politics”, International Studies Quarterly, vol. 47, 2005, pp. 511-531; sur les pratiques des professionnels de la sécurité, voir Bigo D., Polices en réseaux : l'expérience européenne, Paris, Presses de Sciences Po, 1996.

17. La représentation culturelle d'internet comme un monde virtuel miné par la subversion ( " pirates »), l'épidémie (« virus », « propagation », « contamination »), les parasites (« vers »), la fourberie et la ruse ("Cheval de Troie », " porte dérobée ») témoigne du succès du travail symbolique pour sécuriser cette question. Voir Palluault O., "Cybercriminalité et sécurité ", Universalia, 2006, p. 198.

18. Voir sur ce point, MacDonald M., "Securitization and the Construction of Security", 
encore, le renouveau de la défense civile, qui s'incarne après le 11 septembre 2011, s'est installé par étapes successives tout au long des années quatre-vingtdix, sous l'effet de contextes et de stratégies spécifiques et complexes. La sécurisation telle qu'énoncée par Waever n'est qu'un élément; elle n'est qu'un moment de la sécurisation que nous identifions ici. L'acte de parole n'est pour ainsi dire que la partie émergée de l'iceberg que constitue la sécurisation. Il faut surtout veiller à analyser la structuration du champ de la sécurité, les trajectoires et le positionnement des acteurs au sein de ce champ, l'organisation et la distribution des lieux d'autorité du langage sécuritaire, la diffusion et les effets des idées mais aussi des pratiques routinières qui s'élaborent et se développent au sein de ce champ, les relations d'influence réciproque des technologies sur les pratiques de sécurité, les formes d'interpénétration avec d'autres champs issus de la vie sociale ou politique, c'est-à-dire tous les éléments qui vont structurer et influencer l'acte de parole, les autres pratiques non-discursives, mais également les formes et l'issue de leur réception. L'ensemble de ces éléments, qui, en permanence, traversent le champ de la sécurité et le modifient, nous les abordons comme des processus sociaux.

\section{Dynamique et processus sociaux}

Ces processus sont au nombre de cinq. Il s'agit d'abord du processus ontologique qui a trait aux idées, aux images et aux discours sécuritaires qui concourent à la gestion du sens sur la sécurité. Le second est le processus stratégique et renvoie aux relations non discursives entre l'acteur de la sécurité et son objet, notamment les pratiques routinières que sont la collecte et l'analyse des données dans l'appréhension de la menace. Le troisième est le processus sociologique qui vise à cerner les trajectoires des acteurs de la sécurité, les formes de compétition et de jeux qui les relient et les opposent, notamment entre ceux situés dans les lieux légitimés de production du savoir sécuritaire et ceux qui se trouvent à leur marge. Le quatrième processus est le processus bureaucratique. Il consiste en la (re)production des lieux institutionnalisés où l'on élabore, où l'on pratique et où l'on dit la sécurité. Il s'agit principalement de l'organisation de l'État dans le traitement de ses missions de sécurité, mais aussi des effets de concurrence issus de la société civile, notamment des entreprises privées ou des organisations internationales, pour contester à l'État le monopole de faire la sécurité. Le cinquième processus est le processus technologique qui renvoie à l'appréhension des potentiels technologiques, à la fois dans leur contribution à la menace et comme supports de la politique de sécurité.

L'analyse de ces processus et de leurs relations vise à cerner les «petits » changements, souvent invisibles, qui structurent la façon dont va émerger un programme ou une politique qui seront marqués, plus tard et parfois pour des raisons politiques, stratégiques ou financières, du sceau de la nouveauté. On ne prête pas tant d'importance aux formes dominantes des processus, mais 
plutôt aux dissonances, aux phénomènes nouveaux, a priori bénins, mais qui, reliés entre eux, vont contribuer à produire une dynamique et à imposer le changement dans le champ de la sécurité. La sécurisation renvoie en réalité à un phénomène de « dilatation » du champ de la sécurité : la sécurisation (réussie) est l'accomplissement de phénomènes d'expansion du champ de la sécurité et s'incarne à travers la (re)formulation des idées sécuritaires, l'avènement de nouvelles pratiques, l'émergence d'institutions investies d'autorités et de compétences originales, l'avènement de nouveaux acteurs revendiquant une expertise spécifique et l'inflexion des technologies vers des objectifs sécuritaires inédits, ces différents événements intervenant généralement ensemble ${ }^{19}$. Elle se démarque d'une sécurisation avortée qui ne modifie pas l'équilibre du champ de la sécurité 20 .

La démarche engagée ici assume une approche globalisante, qui se distingue des analyses visant à segmenter des phases temporelles comme peuvent le faire les spécialistes des politiques publiques, ou à sectoriser la société pour découvrir les acteurs ou les structures qui agiraient de manière prépondérante sur la constitution de la dynamique de sécurité ${ }^{21}$. En utilisant les concepts de processus et de dynamique, on s'intéresse avant tout à déceler les éléments d'évolution qui se sont installés dans un contexte d'apparente stabilité. Une telle démarche nécessite toutefois quelques précautions. La première est de ne pas aborder les processus comme des secteurs cloisonnés et imperméables, mais à l'inverse, de cerner la manière dont ils s'enchevêtrent et s'interpénètrent. La seconde est de ne pas appréhender les dynamiques de sécurité comme des phases temporelles se succédant dans un ordre chronologique et hiérarchique. Les dynamiques de sécurité apparaissent plutôt à la façon de plaques tectoniques; elles se superposent à des dynamiques en cours, parfois pour se substituer totalement à celles-ci mais plus généralement en cohabitant avec elles pendant une durée très longue ${ }^{22}$. Troisièmement, et par conséquent, une dynamique de sécurité ne saurait être assimilée aux valeurs, idées, représentations et intérêts qui dominent le champ de la sécurité dans un contexte donné. Une dynamique de sécurité ne structure pas l'ensemble du champ de la sécurité, même si elle le modifie. Elle est un élément de changement, pas un nouveau schéma dominant. L'apparition d'une dynamique de la bomeland

European Journal of International Relations, 2008, vol.14, $\mathrm{n}^{\circ} 4$, pp. 563-587, notamment pp. 575-577.

19. La dé sécurisation renvoie alors à un phénomène de rétrécissement du champ de la sécurité, là où Weaver l'appréhende comme le retrait d'une question de l'agenda sécuritaire.

20. Dans ce cas, ce n'est pas que l'on n'observe pas d'évolution des processus sociaux, mais simplement que ces derniers ne produisent pas d'effets convergents, susceptibles de générer une dynamique.

21. Pour une approche de type séquentielle, qui cherche à identifier les différentes phases de maturation d'une politique, voir Kingdon J., Agendas, Alternatives, and Public Policies, New York, Harper Collins, 1984. La sectorisation dans les approches de sécurité a été proposée par Buzan B., People, States and Fear: An Agenda for International Security Studies in the PostCold War Era, New York, Harvester Wheatsheaf, 2nde édition, 1991.

22. Le lien entre dynamique et processus implique qu'une dynamique puisse péricliter en fonction de l'évolution des processus et d'apparition de divergence entre ces derniers. 
security ne signifie donc pas que la politique de sécurité nationale américaine dans sa globalité doive être regardée et comprise à l'aune de cette seule dynamique.

\section{Processus sociaux et transformation sécuritaire dans l'après guerre froide}

La politique de la bomeland security instaurée sous l'administration Bush après les attentats contre le World Trade Center parachève une dynamique commencée au moins une décennie plus tôt. Cette dynamique contemporaine de la sécurité s'est imposée sur les débris de la Guerre froide et le bouleversement à la fois cognitif et matériel qui s'en est suivi, même si ses fondations étaient déjà présentes depuis plusieurs années. Sa construction a reposé sur les mutations convergentes des processus sociaux et a donné naissance au cours des années quatre-vingt-dix au renouveau des politiques de défense civile.

\section{Le processus ontologique : la reconstruction d'une Amérique forte et vigilante}

Le processus ontologique renvoie aux discours de sens, aux idéologies, aux idées, aux schémas interprétatifs, aux symboles qui prédominent au cours d'une période. Il s'agit ici de comprendre les mécanismes de production d'un discours de vérité en prêtant attention à sa construction argumentative, aux effets qu'il provoque sur la population, mais aussi aux stratégies de positionnement qu'il induit et aux nouvelles formes de consensus qu'il génère parmi les « gestionnaires du sens ». Ces derniers sont les acteurs qui détiennent une position sociale à partir de laquelle ils peuvent prétendre donner une interprétation ou une explication des affaires du monde, et d'où est ensuite déduite une représentation particulière de la sécurité : experts, personnalités politiques, universitaires, journalistes, écrivains et romanciers, réalisateurs de films, prédicateurs ${ }^{23} \ldots$

La fin de la Guerre froide s'est imposée comme une période de profond renouvellement des discours de sens à partir de la remise en cause, déjà largement entamée dans les années quatre-vingts, des idées développées par le néoréalisme waltzien ${ }^{24}$. Ce renouvellement a notamment visé le cœur même de la pensée du réalisme structuraliste pour qui la domination d'un seul pays sur tous les autres est impossible du seul fait de l'équilibre de la puissance ${ }^{25}$. Il

23. Nous rappelons que l'analyse de ce processus n'a pas vocation à identifier et mettre à jour de nouveaux discours dominants mais plutôt à saisir les mutations des discours de sens. Cela se remarque notamment à travers l'étude de la parole «savante » à partir des débats qui ont secoué les spécialistes des relations internationales aux États-Unis dans l'après Guerre froide. Toutefois, nous aurions pu opter pour des objets différents, tels que l'analyse des figures de la violence et de la menace dans la filmographie américaine post Guerre froide.

24. Sur l'influence du courant réaliste et les difficultés rencontrées par cette école à la fin des années quatre-vingts, voir Guzzini S., Realism in International Relations and International Political Economy: The Continuing Story of a Death Foretold, London, New York, Routledge, 1998.

25. Selon cette approche, la chute de l'Union soviétique et la fin de la bipolarité ne pouvaient 
s'est imposé en deux temps par une mise à l'écart progressive des positions sceptiques et pessimistes des néo-réalistes vers l'adoption d'idées davantage optimistes, moralistes et nationalistes, dans leur versant libéral ou néoconservateur ${ }^{26}$. Le premier temps a été la démonstration dès les années quatre-vingts de la possibilité de la primauté américaine, avancée à la fois par des auteurs néolibéraux comme Joseph Nye et son concept de "soft power ", ou des réalistes comme Stephen Walt et ses travaux sur la formation des alliances ${ }^{27}$. Le second temps s'est ouvert avec la promotion d'idées davantage normatives qui ne se limitent pas à expliquer en quoi le monde unipolaire est possible, mais indiquent également en quoi il est souhaitable. Auparavant théorisée comme un système dangereux et instable, l'unipolarité est ainsi reconsidérée dans les discussions politiques et académiques américaines comme une configuration bénéfique à la paix et à la prospérité mondiale ${ }^{28}$. Ces thèses émanent principalement du courant libéral, et notamment des travaux sur la paix démocratique ou sur l'institutionnalisme ${ }^{29}$. Elles sont également le fait de néoconservateurs comme Richard Krauthammer, ou plus fondamentalement d'universitaires issus du réalisme comme Wolhforth, qui achèvent de déplacer la ligne principale des conflits académiques et entérinent la marginalisation du néoréalisme waltzien à la fin des années quatre-vingt-dix au profit d'un nouveau consensus sur le sens accordé à la puissance américaine ${ }^{30}$.

engendrer que le retour à des pratiques de rééquilibrage de la puissance amenant les anciens alliés des États-Unis à s'éloigner de sa tutelle, à s'opposer à ses prétentions ou à sa réalité hégémonique, ou encore à contraindre ses actions unilatérales en les insérant dans le cadre des organisations internationales. Voir Waltz K., Theory of International Politics, Reading, Mass.: Addison-Wesley, 1979.

26. Les théories pessimistes sur le déclin américain se sont également imposées en dehors de l'école néo-réaliste. Quelques exemples : Kennedy P., The Rise and Fall of the Great Powers: Economic Change and Military Conflict from 1500 to 2000, New York, Random House, 1987, notamment pp. 489-600; Rosecrance R. (ed.), America as an Ordinary Country, Ithaca, N.Y., Cornell University Press, 1979.

27. Nye J., Bound to Lead: the Changing Nature of American Power, New York, Basic Books, 1990 ; Walt S., The Origins of Alliances, Ithaca, Cornell University Press, 1987. Pour des critiques contemporaines contre l'école décliniste, voir aussi Russett B., "The Mysterious Case of Vanishing Hegemony; or Is Mark Twain Really Dead?”, International Organization, vol. 39, 1985, pp. 207-31; Strange S., "The Persistent Myth of Lost Hegemony", International Organization, vol.41, n4, 1987, pp. 551-574 ; Nau H., The Myth of America's Decline: Leading the World Economy into the 1990s, New York, Oxford University Press, 1987 ; Huntington S., “The U.S. - Decline or Renewal?”, Foreign Affairs, vol.67, n¹, hiver 198889, pp. 76-96.

28. Krauthammer C., "The Unipolar Moment", Foreign Affairs, n70, 1990-91, p. 16.

29. Les thèses sur la paix démocratique expliquent combien la nature démocratique et libérale du régime exerce une contrainte décisive sur les dirigeants politiques par rapport au déclenchement d'un conflit armé, spécifiquement quand l'adversaire se trouve lui-même être une démocratie. Russett B., Grasping the Democratic Peace: Principles for a Post-Cold War World, Princeton, Princeton University Press, 1993, p. 138 ; Diamond L., "Promoting Democracy", Foreign Policy, n87, 1992, pp. 25-46 ; Muravscik J., Exporting Democracy: Fulfilling America's Destiny, Washington, D.C.: AEI Press, 1991 ; Brown M., Lynn-Jones S., Miller S. (eds), Debating the Democratic Peace, Cambridge, Mass, MIT Press, 1996.

30. Wohlforth W., "The Stability of a Unipolar World", International Security, vol.24, n¹, 1999, pp. 5-41. La position de Wohlforth, comme celle qu'incarne déjà Samuel Huntington, participe d'un rapprochement entre une partie des réalistes et l'idéalisme conservateur sur la nécessité de maintenir la primauté américaine. Plus fondamentalement, des débats naissent au milieu des années quatre-vingt-dix sur cette « dégénérescence » du réalisme. Le terme est 
Ce consensus alimente un large processus politique et culturel de légitimation d'une nouvelle interprétation du système international reposant sur la préservation de la primauté acquise par l'Amérique ${ }^{31}$. Les critiques des libéraux, des constructivistes et des postmodernes à l'encontre des idées néoréalistes mettent fin à la marginalisation d'approches hétéroclites davantage outillées pour penser la suprématie pacifique. Elles aboutissent à la désuétude des schémas stratégiques classiques qui réduisaient largement les affaires de sécurité aux seules questions militaires, et amènent au développement de nouveaux programmes de recherche portant sur des thèmes de sécurité auparavant anecdotiques tels que les phénomènes d'immigration, les trafics de drogue, les phénomènes de violence politique, le rôle des groupes ethniques ou religieux... Elles accompagnent au début des années quatre-vingt-dix la promotion des idées stratégiques sur la nécessaire révolution dans les affaires militaires et la prise en compte de nouvelles méthodes de guerre, notamment issues du développement des nouvelles technologies, comme la guerre de l'information ${ }^{32}$.

Au niveau politique, ce consensus entérine une démarche politique partagée par les démocrates comme les républicains autour de la promotion d'une Amérique forte et vigilante, qu'elle soit perçue comme empire bienveillant ou nation indispensable ${ }^{33}$. S'il n'exclut pas les dissensions vigoureuses sur les formes «douces» ou «fortes » que cette primauté implique, ce consensus amène à envisager une nouvelle grande stratégie où il s'agit de se placer en vigilance, d'agir de manière proactive pour empêcher l'émergence d'un concurrent - pas seulement d'un adversaire - et de regarder l'ensemble des risques, même les moins orthodoxes, pour les appréhender comme des menaces potentiellement dangereuses et destructrices. Cette politique s'incarne dès 1992 dans son versant néoconservateur avec le mémorandum Wolfowitz, puis dans sa version libérale sous l'administration Clinton à travers l'accent mis sur les menaces transnationales, qu'ils s'agissent des trafics de drogue ou du terrorisme ${ }^{34}$. Ce consensus politique a modifié la critériologie

emprunté à John A. Vasquez, "The Realist Paradigm and Degenerative vs. Progressive Research Programs: An Appraisal of Neotraditional Research on Waltz's Balancing Proposition”, American Political Science Review, vol.91, n 4, 1997, pp. 899-912 ; voir aussi Moravcsik A., Legro J., "Is Anybody A Realist?”, International security, vol.24, n², 1999, pp. 5-55 et la réponse à cet article par Peter D. Feaver, Gunther Hellman, Randall L.Schweller, Jeffrey W. Taliaferro, William C. Wohlforth, Andrew Moravcsik, Jeffrey W. Legro, "Brother, Can You Spare a Paradigm (or was Anybody ever a Realist?)", International Security, vol.25, ${ }^{\circ} 1,2000$.

31. Ce consensus se révélera une décennie plus tard au cours de la campagne d'Irak à travers la marginalisation des néo-réalistes opposés à l'entrée en guerre des États-Unis.

32. Les débats sur la guerre d'information (information warfare) et la paralysie stratégique qui ont animé les réflexions des experts de la chose militaire et de la stratégie, notamment en 1992, 1993 et 1994, ont largement permis la construction du thème de la protection des infrastructures critiques. Sur ce point, voir Palluault O., Stratégie de la précaution : la construction de la protection des infrastructures critiques aux États-Unis de 1993 à 2003, op.cit. pp.80-108

33. La notion d'empire bienveillant est de Robert Kagan, "U.S. dominance: is it good for the world?", Foreign Policy, n¹11, p. 24-48; celle de nation indispensable est de Madeleine Albright, Interview on NBC-TV “The Today Show” with Matt Lauer, 19 février 1998.

34. Le très controversé Defense Planning Guidance, rédigé en 1992 par le sous-secrétaire à la 
sur laquelle repose la sécurité nationale américaine. Celle-ci n’est plus tournée vers la survie des États-Unis, conçue comme le respect de l'intégrité territoriale des États-Unis à travers une politique de dissuasion, mais comme la capacité de ce pays à affirmer et maintenir sa supériorité dans les différentes sources de puissance que sont le militaire, l'économique, le technologique ou le culturel. Autrement dit, il ne s'agit pas de vaincre un ennemi comme cela pouvait encore être le cas à la fin des années quatre-vingts, mais d'empêcher tout État ou groupe d'États de concurrencer la puissance américaine, la paix et la prospérité mondiale étant supposées être à ce prix ${ }^{35}$. Ajoutée à la rhétorique sur les menaces terroristes, la protection de la primauté américaine constitue bien la marque d'une re-conceptualisation d'un nouveau défi donné à l'Amérique.

Les programmes de défense civile resurgissent dans ce contexte comme des outils permettant de préserver la primauté américaine conçue en termes civilisationnels. Les deux piliers de la défense civile institués en 1996 en sont l'illustration. La protection des infrastructures critiques, qui vise à sécuriser les réseaux des secteurs stratégiques notamment contre les menaces informatiques, est définie comme la protection des systèmes dont la paralysie minerait la sécurité nationale ou économique du pays ${ }^{36}$. Quant à la protection contre des attentats perpétrés avec une arme de destruction massive, son objectif est de préserver l'Amérique d'une catastrophe humaine mais aussi symbolique ${ }^{37}$. Plus largement, les États-Unis s'engagent dans une voie nouvelle de «défense préventive » qui englobe à la fois le renouveau de la défense civile, l'élaboration du programme d'écoute mondial Échelon ou la résurgence du projet de bouclier anti-missile, dont on ne sait pas très bien contre quel ennemi il est supposé protéger l'Amérique ${ }^{38}$. La doctrine de dissuasion se trouve totalement renouvelée, la relation «fort » / « fort », en vigueur contre l'URSS pen-

défense Paul Wolfowitz, appelle très explicitement l'Amérique à prévenir l'émergence d'un rival potentiel. Bien que ce mémorandum ait été retoqué et reformulé en des termes plus conciliants et moins provocateurs, l'esprit qui l'anime imprègne largement la décennie des années quatre-vingt-dix. Voir Tyler P., "U.S. Strategy Plan Calls for Insuring No Rivals Develop A One-Superpower World Pentagon's Document Outlines Ways to Thwart Challenges to Primacy of America", New York Times, 8 mars 1992 ; voir également Gellman B., "Keeping US First: Pentagon Would Preclude a Rival Superpower", Washington Post, 11 mars 1992.

35. Comme l'exprime la première phrase de la stratégie de sécurité nationale de 1998, l'intérêt national américain est assimilé à la paix, à la prospérité et à la démocratie dans le monde : Clinton W., A National Security Strategy for A New Century, Washington DC, The White House, 1998, p. iii.

36. President's Commission on Critical Infrastructure Protection, Critical Foundations, Protecting America's Critical Infrastructures, Washington DC, The White House, octobre 1997, 192 pages.

37. Il s'agit autant de prévenir des victimes que l'inviolabilité du territoire américain. Les attentats du 11 septembre soulignent combien cette dimension symbolique était primordiale, les terroristes voulant marquer l'effondrement du pouvoir financier (World Trade Center) et militaire (Pentagone) américain au moins autant sinon plus que provoquer le plus grand nombre de victimes.

38. Carter A., Perry W., Preventive Defense: A New Security Strategy for America, Washington DC, Brookings Institution Press, 1999. 
dant la Guerre froide, se voyant substituée moins une relation asymétrique «fort » / «faible » qu’à une relation « moi » / « autres », le « moi » étant considéré dans sa dimension culturelle et civilisationnelle.

Le processus ontologique entraîne enfin la réaffirmation discursive et symbolique d'une menace existentielle capable de fonder une identité commune, à qui l'on confère les capacités et les traits moraux qui en font un double négatif de l'Amérique ${ }^{39}$. En dépit des divergences dans leur démarche épistémologique et dans l'imaginaire qu'ils convoquent, la majorité de ces discours ont convergé vers la menace du terrorisme, qui s'est déclinée en plusieurs visages en fonction de l'usage fait par ceux qui l'ont mobilisée : cyberterrorisme, terrorisme chimique, terrorisme nucléaire, terrorisme biologique, agroterrorisme, terrorisme étatique, terrorisme transnational, terrorisme intérieur... Le terrorisme ne se réfère pas à des groupes spécifiques et identifiables mais plutôt à une pluralité d'acteurs sans lien réel. Cette plasticité facilite le travail d'amalgame qui entretient un imaginaire du terrorisme comme menace mondiale et homogène, alors même qu'elle renferme des stratégies, des auteurs, des méthodes dissemblables et souvent antagonistes 40 .

\section{Le processus stratégique : la substitution de la vulnérabilité à la menace comme pivot du travail stratégique}

Le processus stratégique traite des pratiques des acteurs de la sécurité eu égard à leur objet de travail. Il s’intéresse à la manière dont ils appréhendent «scientifiquement » le danger, dont ils représentent et construisent socialement la menace. Il s'agit moins de prêter attention aux discours de sécurité qu'au travail sécuritaire proprement dit, dans sa réalité concrète, quotidienne et routinière, ou plutôt de cerner comment la production du langage sécuritaire est intimement dépendante de la production d'un «savoir » et d'une technique sécuritaires. La fin de la Guerre froide introduit ici un changement assez brutal, et pourtant largement sous-estimé, dans la manière d'appréhender la menace de la part des spécialistes de la sécurité : à l'approche traditionnelle et dominante, que l'on peut qualifier de déductive se substitue une approche inductive, déjà abordée à partir des années soixante-dix en matière de gestion des risques ${ }^{41}$.

39. Sur la construction culturelle de l'ennemi, voir Weldes J., Laffey M., Gusterson H. (eds.), Cultures of Insecurity: States, Communities, and the Production of Danger, op.cit.

40. Une fois encore, l'analyse des figures de l'ennemi dans les productions cinématographiques hollywoodiennes de l'après Guerre froide aboutit à la même conclusion. Elle éclaire comment s'est imposée la figure du terroriste sous des traits divers et flous, en substitution de figures plus classiques davantage structurées par les enjeux de la Guerre froide. Voir Boggs C., Pollard T., "Hollywood and the Spectacle of Terrorism", New Political Science, vol.28, n³, septembre 2006, pp. 335-351.

41. Sur l'imprégnation d'une culture du risque en matière d'anti-terrorisme et de gestion des menaces, voir l'ouvrage collectif dirigé par Louise Amoore et Marieke de Goede, Risk and the War on Terror, New York, Routledge, 2008. 
Traditionnellement, l'objet stratégique de référence est la menace, ellemême étant la conjonction entre une capacité destructrice et la volonté réelle de l'utiliser. Cette menace est toujours rattachée à un sujet spécifique et identifié, généralement un État dans le cas des études stratégiques classiques ${ }^{42}$. Avec la disparition de l'ennemi communiste - mais pour des raisons qui dépassent la fin de la Guerre froide - la menace est remplacée par la vulnérabilité comme objet structurant les pratiques stratégiques. Pour dire autrement, le regard des experts s'est porté non plus vers des sources extérieures de danger, présentées désormais comme imprévisibles, mais sur les faiblesses internes de la société, identifiées à partir du travail d'expertise du risque (risk management) ${ }^{43}$. Les travaux de la communauté de la défense et du renseignement pour repenser la sécurité dans le nouveau contexte post-Guerre froide témoignent de cette imprégnation par les acteurs de la défense des modèles d'appréhension du risque de plus en plus élaborés et appliqués par des experts civils dans le cadre de la prévention des catastrophes naturelles ou technologiques ${ }^{44}$. Tandis que dans l'approche déductive, la vulnérabilité procède (est "déduite ») de la connaissance, réelle ou supposée, de la menace, elle devient dans l'approche inductive le pivot de la pratique stratégique à partir de laquelle on reconstruit la figure de la menace ${ }^{45}$. La démarche inductive efface la distinction entre la possession ou la maitrise d'une arme et son usage imminent, sans considération sur l'acteur stratégique qui en est l'auteur ${ }^{46}$. Elle aboutit à la position prise par la commission présidentielle sur la protection des infrastructures critiques en 1997 qui appelle à assumer une nouvelle appréhension de la menace, une appréhension « tournée vers la seule capacité à détruire, plutôt que vers l'identification de la personne, du groupe ou de la nation disposés à utiliser une telle capacité 47 . » Cette démarche fait sens sitôt que s'impose le postulat de l'incapacité à éprouver l'ennemi avant qu'il ne frappe, ou comme le dirait Ülrich Beck, à partir du moment où « l'on en est réduit à évaluer les dangers à l'aide d'instruments de mesure, de théories, et surtout d'absence de connaissance ${ }^{48}$. » Elle est par ailleurs présente dès le

42. Pour une définition de la menace, on s'appuie sur les travaux de Stephen Walt, The Origins of Alliances, op.cit.

43. La démarche déductive a demeuré au sein des études stratégiques dans le cadre du volet "rogue states» afin d'identifier la dangerosité des États voyous, avec des difficultés toutefois pour recréer une figure de l'ennemi « crédible ». Sur ce point, voir Badey T., "US Anti-terrorism Policy: the Clinton Administration”, Contemporary Security Policy, vol.19, n², 1998, pp. 50-70.

44. Voir Joint Security Commission, Redefining Security: A Report to the Secretary of Defense and the Director of Central Intelligence, Washington DC, 28 février 1994, p. iv.

45. La démarche réaliste invite à faire de la vulnérabilité un facteur dépendant de la menace. Elle n'appréhende pas l'invulnérabilité comme une situation d'indestructibilité; elle ne signifie pas qu'il n'existe pas des failles, des zones de risque, mais plutôt que ces failles ne dérivent pas d'un acteur stratégique.

46. Voir par exemple Defense Science Board, Report of the Defense Science Board Task Force on Information Warfare - Defense, Washington DC: Department of Defense, Office of the Undersecretary of Defense for Acquisitions and Technology, novembre 1996, p. 6-27.

47. President's Commission on Critical Infrastructure Protection, Critical Foundations: The Report of the President's Commission on Critical Infrastructure Protection, op.cit., p. 20.

48. Beck U., La société du risque : sur la voie d'une autre modernité, Paris, Aubier, 2nde édition, 
milieu des années quatre-vingt-dix au sein des services de renseignement américains qui annoncent aborder un nouveau " paradigme de la menace », dans lequel il est assumé que l'ennemi est désormais inconnu ${ }^{49}$. Cet effacement de la menace dans les pratiques stratégiques ne doit donc pas être perçu comme le résultat de la disparition de l'Union soviétique : il n'est pas plus le fait d'un constat objectif que d'une posture naïve. Il relève non pas d'un élément conjoncturel, mais plutôt de l'imprégnation d'une démarche réflexive au sein même des pratiques stratégiques, qui aboutit à reconnaître l'impossibilité d'identifier et d'éprouver son adversaire dans un contexte d'incertitude.

Si la menace ne constitue plus un élément structurant les pratiques stratégiques, la présence d'une figure de l'ennemi demeure incontournable, pour des raisons de crédibilité politique et stratégique. Elle est toutefois recréée à partir des vulnérabilités identifiées au sein même de la société américaine, et se voit conférée les capacités destructrices et l'intention malveillante. L'analyse des jeux de guerre éclaire la façon dont la figure de l'ennemi apparaît de plus en plus imprécise et floue, à mesure que les acteurs qui élaborent les scénarios investissent dans cette figure, non plus seulement leurs savoirs, leurs connaissances, leurs opinions, mais aussi leurs doutes et leurs ignorances ${ }^{50}$... Mieux, la représentation de la menace, notamment du cyberterrorisme, tient largement de cette posture réflexive, que nous avons explicitée plus haut : elle est liée, non pas à l'existence réelle d'un adversaire prêt à attaquer l'Amérique avec des escadrons d'experts en informatique, mais à la crainte que des pays disposent bientôt des mêmes capacités et des mêmes technologies que les États-Unis sont en train de développer pour s'assurer la suprématie de l'information ${ }^{51}$.

2001, p. 15.

49. Voir l'audition du directeur de la National Security Agency devant le sénat américain: Hughes P., "Global Threats and Challenges to the United States and its Interests abroad", Statement For The Senate Select Committee On Intelligence, 5 février 1997.

50. Palluault O., Stratégie de la précaution : la construction de la protection des infrastructures critiques aux États-Unis de 1993 à 2003, op.cit., pp. 186-187.

51. Patrie de l'internet et du développement des réseaux cybernétiques, l'Amérique n'est pas seulement la cible idéale de la guerre d'information, encore est-elle, en ce milieu des années quatre-vingt-dix, le principal sinon le seul véritable belligérant de cette guerre. Elle constitue l'archétype de l'adversaire pouvant mener une offensive d'information visant à détruire les infrastructures critiques d'un État. Elle seule dispose réellement à la fois des capacités et de la volonté de détruire les infrastructures critiques d'un État. Les premières sont mises en place et testées dans le cadre de programmes secrets développés au sein de la National Security Agency, de la Defense Information Systems Agency et d'autres organismes spécialisés dans la guerre d'information au sein du département de la défense dès les années quatre-vingts. La seconde est officialisée et légitimée dans les doctrines et les autres documents de stratégie militaire. Dès 1998, le département de la Défense reconnaît ainsi les Computer Network Operations comme une pratique de guerre. 

sécurité nationale

Le processus sociologique nous éclaire sur les acteurs de la sécurité, sur leurs itinéraires, leurs trajectoires sociales, sur les jeux de pouvoirs qu'ils entretiennent et les compétitions sociales qu'ils nourrissent. Son analyse nous révèle comment émerge vers la fin de la Guerre froide une nouvelle figure de l'expert en sécurité nationale, qui va concurrencer - et dans certains cas se substituer à - la figure traditionnelle. Ce nouvel expert se distingue du précédent en ce qu'il dispose d'un bagage plus technique que géopolitique, et sa compétence première est l'identification des vulnérabilités, non la compréhension des menaces. C'est avant tout un civil, spécialiste du risque, qu'il soit informatique, naturel, sanitaire ou technologique ${ }^{52}$. Assez éloigné de la figure du militaire ou du diplomate, il est généralement dépourvu d'une connaissance approfondie de la stratégie et des études de sécurité 53 . Toutefois, en accordant à son objet un caractère résolument sécuritaire, cet acteur investit le champ de la sécurité en prétendant au monopole de la connaissance scientifique en matière de sécurité nationale. On voit ainsi au milieu des années quatre-vingt-dix un certain nombre d'experts en sécurité informatique se définir comme des spécialistes du cyberterrorisme, investir les think tanks, populariser leurs écrits et leurs idées à partir d'internet, publier des articles dans des revues de sécurité nationale et s'imposer grâce à des opérations de dramatisation sur un prochain «Pearl Harbor électronique » 54 . Auparavant marginalisés par rapport aux institutions légitimes en matière de production du savoir sécuritaire, ces acteurs interviennent peu à peu dans les enceintes universitaires ou médiatiques pour parler de la menace informatique, jusqu'à s'imposer aux côtés des spécialistes issus de la communauté du renseignement ou de la défense. Le succès de ces entreprises, renforcé et crédibilisé par les mesures

52. Les travaux de la protection des infrastructures critiques ont largement reposé sur des ouvrages issus de la sécurité informatique, notamment National Academy of Sciences, Computer science and telecommunications Board, Computers at Risk: Safe Computing in the Information Age, Washington DC: National Research Council, 1991, 303 p. Sur la bibliographie utilisée pendant les deux ans de travail de la commission présidentielle, voir The President's Commission on Critical Infrastructure Protection, Open Source Bibliography, Washington DC : CPPIC, 1997, 71 pages.

53. Par exemple, il est notable que les écrits portant sur le cyberterrorisme et la menace aux infrastructures critiques ne s'insèrent jamais dans les débats académiques sur la sécurité nationale et l'utilisation de la violence politique. De la même manière, les universitaires et les experts « en place» ont largement boudé ces sujets.

54. L'un des exemples est notamment Dorothy Denning, initialement professeur en sécurité informatique à l'université Georgetown, devenue après ses écrits sur le cyberterrorisme, professeur au sein de l'école militaire navale de Monterey en Californie, ou James Adams ancien journaliste devenu entrepreneur en sécurité informatique puis conseiller au sein du National Security Agency à la faveur de ses prises de position contre le cyberterrorisme. Voir notamment Denning D., "Is Cyber Terror Next?”, in Calhoun C., Price P., and Timmer A. (eds), Understanding September 11, New York, The New Press, 2002, pp. 191-197 ; Adams J., "Virtual Defense”, Foreign Affairs, vol.80, n³, mai-juin 2001, p. 98-112 ; sur l'expression "Electronic Pearl Harbor », voir Smith G., "An Electronic Pearl Harbor? Not Likely”, Issues in Science and Technology, vol.15, $\mathrm{n}^{\circ} 1,1998$, pp. 68-73. 
gouvernementales sur la protection du territoire et la défense civile, a permis au discours sur le cyberterrorisme ou sur la menace des armes de destruction massive de s'institutionnaliser, et de démultiplier les opportunités sociales et professionnelles pour ceux qui s'en réclament les spécialistes 55.

Mais le réel changement consiste moins en quelques trajectoires sociales réussies que dans le mouvement général des acteurs de la sécurité vers la mobilisation d'un certain type de savoir, plus technique et moins géopolitique, et vers la réorientation de leurs discours pour intégrer les préoccupations sur les risques informatiques, technologiques ou sanitaires au prisme de la lutte contre le terrorisme. Les spécialistes du terrorisme pendant la Guerre froide se réinventent experts en cyberterrorisme, ceux travaillant sur la prolifération nucléaire à la chute de l'Union soviétique adaptent leurs connaissances pour traiter de la menace posée par l'utilisation des armes de destruction massive par des groupes transnationaux ${ }^{56}$. Certaines personnalités haut placées dans l'administration Clinton en matière de sécurité nationale, comme John Deutch, ancien directeur de la CIA ou Ashton Carter, conseiller du secrétaire à la Défense sur la politique de sécurité internationale, reprennent à leur compte les thèses sur le nouveau terrorisme, pour mieux les crédibiliser et les populariser ${ }^{57}$. Ces exemples témoignent combien l'avènement d'une nouvelle culture sécuritaire s'accompagne de la mise en avant d'un certain profil d'expertise de la sécurité, éloigné des figures du soldat et du diplomate.

\section{Le processus bureaucratique : la naissance d'une administration moderne de défense civile}

Le processus bureaucratique est relatif aux modes d'organisation de l'État dans l'exécution et le traitement des missions de sécurité nationale. Nous nous intéressons ici à la naissance au cours des années quatre-vingt-dix d'une administration de la défense civile qui accompagne la destruction progressive de l'équilibre institutionnel apparu au cours de la Guerre froide en matière d'anti-terrorisme, qui a ainsi ouvert la voie à la construction du département de la homeland security en 2003. Au sortir de la Guerre froide, l'anti-terro-

55. Les principaux think tank américains ont établi dès la fin des années quatre-vingt-dix des départements spécialisés sur la homeland security. Des centres de recherches privés sont créés sur le cyberterrorisme comme le Terrorism Research Center fondé en 1996, le Potomac Institute for Policy Studies créé en 1994, ou le ANSER Institute for Homeland Security créé quelques mois avant le 11 septembre 2001.

56. Concernant le cyberterorisme, citons notamment Yonah Alexander, auteur de la thèse sur la «Soviet Connection » faisant de Moscou l'origine du terrorisme au cours des années quatrevingts, battue en brèche et contredite avec la fin de la Guerre froide. On trouve également le professeur Walter Laqueur, l'un des premiers à populariser au début des années quatre-vingtdix la crainte du terrorisme technologique. Voir Alexander Y., Swetnam M. (eds.), Cyberterrorism and Information Warfare: Threats and Responses, Ardsley, New York, Transnational Publishers, 2001 ; Laqueur W., The New Terrorism: Fanaticism and the Arms of Mass Destruction, New York, Oxford University Press, 1999.

57. Carter A., Zelikow P., Deutch J., “Catastrophic Terrorism: Tackling the New Danger”, Foreign Affairs, vol.77, n6, 1998, pp. 80-94. 
risme américain relève de l'État fédéral et repose sur les lead agencies, dont les champs de compétences sont séparés par les distinctions menace intérieure (département de la Justice) / menace extérieure (secrétariat d'État), et menace à la sécurité intérieure (FBI) / menace à la sécurité nationale (département de la Défense) ${ }^{58}$. Au milieu des années quatre-vingt-dix, ce modèle s'est transformé sous l'effet conjugué de trois mouvements.

Le premier est l'expansion de l'appareil d'État liée à l'intégration progressive, sous la coupe des agences de sécurité, de compétences "marginales ", c'est-à-dire situées aux marges de leur autorité traditionnelle. Avec la mobilisation et la revendication de nouveaux types d'expertise autour du danger informatique ou des armes de destruction massive, on assiste alors soit à la création de nouvelles structures chargées du traitement de nouveaux objets politiques, soit à l'extension des administrations existantes - celles-ci cherchant à repousser les limites de leur domaine d'intervention -, soit encore aux effets conjugués de ces deux phénomènes ${ }^{59}$. Tandis que le département d’État perd de son autorité en matière d'anti-terrorisme, les départements de la Défense et de la Justice renforcent largement leur emprise, le premier en matière de prévention des attaques avec armes de destruction massive, le second en matière de sécurité intérieure, qu'il s'agisse du danger sur les infrastructures critiques, de cyberterrorisme ou de prévention du terrorisme domestique ${ }^{60}$.

Le second mouvement est la dissémination des lieux d'autorité et de pouvoir au sein de l'appareil d'État. Celle-ci s'opère sous l'effet de l'inflation des organismes participant à la sécurité nationale, notamment au travers des revendications émises par les ministères techniques comme les départements de la Santé, du Commerce ou de l'Énergie. ${ }^{61}$ En 2000, un rapport de la cour des comptes américaine recense pas moins d'une quarantaine d'agences participant à l'anti-terrorisme, revendiquant à ce titre une expertise spécifique et des budgets sans cesse croissants ${ }^{62}$. Cette dissémination intervient également

58. Les deux autres lead agencies sont la Central Intelligence Agency (CIA) pour le renseignement et la Federal Emergency Management Agency (FEMA) pour la gestion des conséquences d'une attaque sur le sol américain.

59. On trouve par exemple la création en 1998 du National Infrastructure Protection Center (NIPC), créé au sein du FBI et du Critical Infrastructure Assurance Office (CIAO) au sein du département du Commerce.

60. La perte d'influence du département d'État est symptomatique de l'utilisation au cours de la Guerre froide de la menace du terrorisme international par la diplomatie américaine comme un outil de déstabilisation du bloc soviétique. La fin de la Guerre froide, et la montée d'un terrorisme domestique en 1993 avec le premier attentat contre le World Trade Center et surtout l'attentat d'Oklahoma City de 1995, ont abouti à un traitement sécuritaire, renforçant de fait la mainmise du département de la Justice, et en son sein, du Bureau Fédéral d'Investigation (FBI).

61. Dans le cadre de la protection des infrastructures critiques, les ministères techniques acquerront dès 1998 la charge de défendre eux-mêmes leurs propres systèmes et de coordonner le renforcement des mesures de protection dans leur domaine d'activité en lien avec le secteur privé.

62. Voir notamment General Accounting Office, Combating Terrorism: Issues in Managing 
à travers les phénomènes de spécialisation et de transversalité qui touchent l'administration. Les premiers accordent une place majeure aux réseaux d'experts, aux divers organismes de conseil, et entraînent une collaboration plus aiguë entre les sphères publiques et privées ${ }^{63}$. Les seconds assurent un décloisonnement administratif qui casse un système basé sur une configuration hiérarchique pour lui substituer une organisation plus complexe, à l'image du rhizome ${ }^{64}$. Par ailleurs, la structure fédérale américaine accroît une transversalité verticale entre les organismes dépendants de l'État central et les diverses structures relevant soit des États fédérés, soit des autorités locales (communales notamment).

Enfin le troisième mouvement est relatif au renforcement des systèmes de coordination surplombant les administrations principales (lead agencies). Ce mouvement de transfert du pouvoir depuis des agences exécutives vers des structures plus informelles, plus souples, mais également dépourvues d'attribution de gestion, a modifié considérablement les équilibres institutionnels auparavant centrés sur la neutralisation réciproque des agences principales. Ce mouvement a d'abord privilégié des organes interministériels comme le National Security Council. C'est en son sein qu'est établi en 1998 le bureau du Coordinateur national pour le contre-terrorisme, la protection des infrastructures et la sécurité, chargé de superviser et de coordonner les actions en matière d'anti-terrorisme et de défense civile ${ }^{65}$. Ce bureau devient avant le 11 septembre 2001 le point focal de la politique anti-terroriste et assure notamment la promotion du volet autour de la protection des infrastructures critiques et du cyberespace. Ce mouvement de renforcement des structures de coordination, lié au délitement de l'équilibre institutionnel issu de la Guerre froide, aboutit à la tentation de concentration, c'est-à-dire au transfert de compétences exécutives à ces systèmes de coordination, dont la création du département de la bomeland security en janvier 2003 constitue la parfaite illustration.

Counterterrorist Programs, Washington DC, General Accounting Office, 6 avril 2000 ; General Accounting Office, Combating Terrorism: Observations on Growth in Federal Programs, Statement of Mark E. Gebicke before the Subcommittee on Oversight, Investigations, and Emergency Management, Committee on Transportation and Infrastructure, House of Representatives, Washington DC, General Accounting Office, 9 juin 1999.

63. Les institutions de coordination entre le public et le privé se sont multipliées dans le domaine de la sécurité nationale, et notamment en matière de défense civile. En plus du National Security Telecommunications Advisory Committee (NSTAC), fondé en 1982, et chargé de conseiller le président des États-Unis sur la politique de sécurité relative aux télécommunications, est créé en 1998 le NIAC (National Infrastructure Assurance Council), un organisme consultatif du Président où siègent les représentants du secteur privé. Surtout, la politique de protection des infrastructures critiques institue les ISACs (Information Sharings and Analysis Centers), des structures privées qui se voient chargées dans chacun des grands secteurs stratégiques de la mise en place de centres d'analyse et de partage de l'information sur les menaces.

64. Les nouvelles structures créées dans le cadre de la protection des infrastructures critiques sont largement inter-ministérielles, et visent avant tout un travail de coordination de multiples agences.

65. Clinton W., Presidential Directive Decision 62: Combating Terrorism (white paper), Washington DC, The White House, 22 mai 1998. Le document dans son entier est classé. 
Le processus technologique est lié au développement des technologies d'information et de communication et à leur intégration dans le fonctionnement quotidien de la société. Ce processus ne saurait toutefois s'assimiler à un simple phénomène matériel. Il correspond à une pensée et à des pratiques spécifiques qui placent les technologies d'information à la fois comme la source des nouvelles vulnérabilités et le facteur indispensable à leur solution. La fin de la Guerre froide a coïncidé avec l'avènement des nouveaux médias et des " autoroutes de l'information » que constitue internet ${ }^{66}$. En quelques années, les opérations quotidiennes des infrastructures du pays, y compris en matière de contrôle et de sécurité, ont été pilotées automatiquement à partir d'ordinateurs, eux-mêmes gérés à distance à travers des réseaux informatiques, générant la crainte de la part des experts militaires américains que « notre puissance économique et militaire repose sur des fondations technologiques que nous ne contrôlons pas et que $[\ldots]$ nous ne comprenons pas ${ }^{67}$. » Ce n'est pas la technologie en elle-même qui est remise en cause, mais plutôt la croyance en la capacité technologique comme support de croissance, sans que soient pris en considération ses répercussions potentiellement néfastes. Il en va de même dans le plan national de protection des infrastructures critiques établi en 2000 qui souligne que la vulnérabilité des États-Unis est apparue à partir du moment où ce pays est devenu dépendant du fonctionnement des systèmes informatiques sans s'être préalablement interrogé sur la protection de ces systèmes ${ }^{68}$. La technologisation de la société, si elle suscite la fantasmagorie sur le péril informatique, n'est pas soumise à une critique radicale. Au contraire, elle s'incarne dans une technologisation de la sécurité à travers la généralisation des systèmes d'identification, de contrôle et de surveillance, non seulement verticalement aux différents niveaux de la société (gouvernement, États fédérés, entreprises, individus), mais également horizontalement, au niveau des différents secteurs d'activités de la société : on voit ainsi comment la création de banques de données informatiques, le développement de la vidéo-surveillance dans les espaces publics, l'utilisation de la biométrie, des puces électroniques, les écoutes généralisées à partir de systèmes gérés par satellites deviennent les nouveaux outils de la lutte anti-terroriste, développés par des firmes privées que le gouvernement associe étroitement à la défense du pays ${ }^{69}$.

66. Il faut rappeler qu'initialement s'impose au sujet d'internet une vision optimiste et libertaire appréhendant cet outil comme l'élaboration progressive d'un « village global » tournée vers le partage numérique de la connaissance, et vers l'émancipation tant individuelle que collective par l'inculcation d'un savoir autrement interdit ou inaccessible.

67. Defense Science Board, Report of the Defense Science Board Task Force on Information Warfare-Defense, Washington DC: Department of Defense, Office of the Undersecreatry of Defense for Acquisitions and Technology, novembre 1996, p. 2-3.

68. Clinton W., Defending America's Cyberspace: National Plan for Information Systems Protection Version 1.0: An Invitation to a Dialogue, Washignton DC, the White House, 2000, executive summary, p. vii.

69. Ce rapport du technologique et du sécuritaire, et ses effets sur les libertés publiques, font l'objet de nombreuses recherches ces dernières années. Voir notamment Ceyhan A., « Enjeux 
Le processus technologique souligne ainsi le mouvement perpétuel de sécurisation par la technologie de failles introduites par d'autres technologies. Ce faisant, il renforce le processus sociologique que nous avons identifié plus haut en approfondissant continuellement le besoin d'expertise technologique dans la sécurité nationale, notamment de la part des spécialistes de la sécurité informatique. Loin de représenter une variable matérielle, le processus technologique puise dans un système de croyance qui renvoie au projet moderne des sociétés développées basé sur le progrès technique. À ce titre, le processus technologique est intrinsèquement lié aux discours sur la préservation du mode de vie américain, ce dernier étant défini à partir d'une vision de la sécurité nationale dans laquelle la défense des intérêts nationaux rejoint l'impératif du maintien de la prospérité économique ${ }^{70}$.

\section{La dynamique sécuritaire contemporaine: du renouveau de la défense civile à la homeland security}

Ces cinq processus éclairent chacun un des aspects de la formation du renouveau de la défense civile. Toutefois, pris isolément, ils ne sont pas la cause de la transformation de la sécurité. L'appréciation d'une dynamique de sécurité s'opère nécessairement par l'analyse de l'enchevêtrement des différents processus et de leur convergence. La transformation contemporaine de la sécurité apparaît ainsi à partir de ces lieux de rencontre entre les diverses évolutions du langage, des pratiques stratégiques, l'appréhension de la technologie, le comportement des acteurs et l'organisation des administrations de l’État. Ce sont ces points de rencontre qui dessinent la dynamique.

\section{Analyser les phénomènes d'interactions}

Les points d'entrechoquement des divers processus se sont opérés tout le long des années quatre-vingt-dix. L'analyse vise à soupeser la façon dont ce choc aboutit à la création d'une convergence ou d'une divergence, c'est-à-dire si les évolutions des processus se renforcent mutuellement ou, à l'inverse, s'opposent ou s'annulent. Bien que nous ne puissions présenter un panorama complet des points de rencontre qui ont structuré la dynamique de la bomeland security, voici quelques exemples qui soulignent comment les processus se recoupent et s'influencent les uns les autres.

Un point de convergence apparait au début de l'administration Clinton avec la création du Security Policy Board (SPB). Cet organisme est créé en septembre 1994 et succède à la Joint Security Commission, un comité de liaison

d'identification et de surveillance à l'heure de la biométrie », Cultures $\mathcal{E}$ conflits, nº4, 2006, pp. 33-47.

70. C'est ainsi qu'est redéfinie la sécurité nationale sous l'administration Clinton. Voir Clinton W., National Security Strategy for A New Century, Washington DC, The White House, octobre 1998, p. 5. 
entre le département de la Défense et les agences du renseignement mis en place l'année précédente pour appréhender les enjeux de sécurité de l'après Guerre froide. Dans son rapport de février 1994, la Joint Security Commission consacre à la fois l'idée d'un changement brutal de la sécurité nationale avec la fin de la Guerre froide mais également la nécessité de « développer un nouveau système de sécurité [nationale] qui puisse permettre de faire face aux défis qui vont émerger dans les dernières années de ce siècle et au début du siècle prochain ${ }^{71}$. » Ce rapport est l'un des premiers documents du gouvernement américain de l'après Guerre froide qui assume la disparition de la menace et l'avènement de la gestion du risque dans la sécurité nationale ${ }^{72}$. Il est aussi parmi les premiers à placer la sécurité des systèmes d'information comme un enjeu majeur de la sécurité nationale. Le Security Policy Board, créé au sein du Conseil de sécurité nationale (National Security Council) sur recommandation du rapport de la Joint Security Commission, est l'instrument de diffusion et de mise en place de cette vision sécuritaire ${ }^{73}$. Il est aussi un nouvel outil d'organisation au sein du gouvernement car, composé initialement des seules agences de la défense et du renseignement, il comprend dès septembre 1994 des représentants des ministères civils de l'énergie, du commerce, du budget et de la justice ${ }^{74}$. Ne parvenant pas à assurer une coordination efficace entre les différents niveaux de hiérarchie ni à éviter que les décisions prises ne se résument à un «consensus mou ", le Security Policy Board dérive, dès la fin de l'année 1995 , vers une structure de plus en plus inutile ${ }^{75}$. Pour autant, son impact sur l'émergence de la problématique de la bomeland security n'est pas mineur et doit être mesuré à l'aune de plusieurs évolutions qu'il a profondément accompagnées et renforcées. Tout d'abord, le travail d'expertise effectué en son sein a contribué à mieux structurer les propositions visant à protéger les systèmes informatiques civils considérés comme stratégiques. Ensuite, le SPB a validé l'intégration directe des agences civiles et techniques dans le traitement de questions sécuritaires comme la protection des systèmes d'information. Par ailleurs, il constitue l'une des premières illustrations de la composition au sein de l'administration américaine d'une fracture entre les « agences sécuritaires » et les « agences non sécuritaires » qui se substitue au rapport de force entre les agences de sécurité nationale d'un côté et les agences civiles de l'autre ${ }^{76}$.

71. Ce rapport sanctionne également la désuétude du système de sécurité américain dans le contexte post Guerre froide. Joint Security Commission, Redefining Security: A Report to the Secretary of Defense and the Director of Central Intelligence, Washington DC, 28 février 1994, p. iii.

72. Ibid, p. 2-3.

73. Le Security Policy Board a été établi sur décision présidentielle. Voir William J. Clinton, Presidential Directive Decision 29: Security Policy Coordination, Washington DC: Office of the Press Secretary, 16 septembre 1994.

74. A travers ses divers comités, le SPB regroupe près de 35 agences.

75. Le SPB a été largement critiqué, notamment par la commission Moynihan (Commission on Protecting and Reducing Government Secrecy) pour son inefficacité et son manque de transparence. Un aperçu des critiques est donné dans Lardner R., “Keeping Secrets”, Government Executive, vol.30, n³, mars 1998, pp. 27-29.

76. Cette collusion s'est déjà remarquée dans le cas de l'affaire Clipper, du nom d'un crypto processeur élaboré par la National Security Agency en 1988, et mis en service quelques mois après 
Surtout, les recommandations de cet organisme préfigurent la création d'un nouvel espace de sécurité nationale, placé en dehors de la mainmise directe des militaires ou des policiers. Un livre blanc sur la sécurité de l'information émanant du SPB et daté de décembre 1995 propose ainsi différentes solutions pour doter le gouvernement d'une structure bureaucratique responsable de l'identification et du traitement des vulnérabilités et des menaces cybernétiques 77 . Faisant référence à l'incapacité des militaires à imposer leur point de vue dans le cadre d'institutions perçues comme illégitimes, car défendant de manière trop visible les intérêts et les perceptions des agences issues du département de la Défense, ce document promeut la mise en place d'un « organisme impartial au sein de la branche exécutive, et qui soit compétent techniquement et doté de ressources et d'une autorité suffisantes », pouvant équilibrer « les intérêts légitimes mais parfois antagonistes relevant de la sécurité nationale, de la police et de la justice, du commerce et du respect de la vie privée ${ }^{78}$. »

Un deuxième exemple de la convergence des processus s'incarne dans les différents forums bureaucratiques institués par l'administration américaine pour traiter de la révolution informatique ${ }^{79}$. Si le Security Policy Board peut s'apparenter à un forum sécuritaire, les forums bureaucratiques regroupent plus largement les structures liées à l'Information Infrastructure Task Force (IITF), créé début 1993 afin de " travailler avec le congrès et le secteur privé en vue de développer les mesures requises pour accélérer le déploiement d'une infrastructure nationale d'information 80 ». Organisée autour de différents comités et cercles de réflexion présidés par des ministres ou des directeurs des grandes agences publiques, l'IITF a constitué jusqu'en 1995 un puissant élan pour la diffusion des réseaux informatiques. Mue initialement par une vision idyllique du développement de ces réseaux, l'IITF a peu à peu adopté une posture plus sceptique sous l'influence de groupes de travail situés en son sein comme le Security Forum Issues ou le Reliability and Vulnerability Working Group (RVWG) ${ }^{81}$. Influencés par les agences de sécurité, ces groupes de travail ont assuré, parallèlement au Security Policy Board, la diffusion d'une

l'arrivée au pouvoir de Bill Clinton. Voir William J. Clinton, Presidential Directive Decision 5: Public Encryption Management, Washington DC, The White House, Office of the Press Secretary, 16 avril 1993.

77. Security Policy Board, White Paper on Information Infrastructure Assurance, décembre 1995 [http://www.fas.org/sgp/spb/whitepap.html]

78. Ibid.

79. La notion de forum employée ici se distingue de celle de "forum hybride » employé par Pierre Lascousmes, Yannick Barthe et Michel Callon en ce qu'elle se réfère à des organismes davantage institutionnalisés par le pouvoir que dérivant d'une conflictualité. En revanche, elle s'en rapproche en ce qu'elle met aux prises des experts et des profanes (souvent des fonctionnaires). Voir Lascousmes P., Callon M., Barthe Y., Agir dans un monde incertain : Essai sur la démocratie technique, Paris, Seuil (la couleur des idées), 2001.

80. Sur l'organisation et les travaux de l'Infrastructure Information Task Force, voir Gore A., National Information Infrastructure: Agenda for Action, Washington DC, The White House, 15 septembre 1993.

81. IITF Security Policy Forum, NII Security: The Federal Role, Washington DC: Office of Management and Budget, draft version, 1995, 52 pages. 
vision sécuritaire de la révolution technologique et son inscription sur l'agenda politique en tant que problème de sécurité nationale. Ces forums ont également permis à des experts en sécurité informatique ou à des spécialistes de la gestion des risques de côtoyer des acteurs de la sécurité de l'administration américaine, de les influencer sur les nouvelles sources de la vulnérabilité américaine. La participation d'acteurs privés, impliqués dans des entreprises de sécurité informatique, a aussi joué dans la promotion d'outils technologiques contre les attaques informatiques. Enfin, elle a entraîné l'intégration des ministères techniques dans la résolution des problèmes de sécurité nationale en leur accordant un rôle dans la mise en œuvre de certaines compétences.

La création de la commission présidentielle sur la protection des infrastructures critiques en juillet 1996, et les suites données aux travaux de cette commission, constituent un troisième point de convergence ${ }^{82}$. S'appuyant sur les travaux élaborés depuis plusieurs années au sein des forums bureaucratiques que nous avons évoqués plus haut, la commission présidentielle a légitimé l'approche sécuritaire de la révolution informatique en assurant le couplage décisif entre la lutte contre le terrorisme et la prévention des risques informatiques. Elle a alimenté les théories sur le cyberterrorisme et l'avènement d'un prochain «Pearl Harbor électronique », crédibilisant ainsi le discours et les intérêts des acteurs agitant cette peur ${ }^{83}$. Rendant son rapport en novembre 1997, la commission présidentielle a joué un rôle prépondérant auprès de l'opinion publique et le secteur privé pour justifier l'intervention du gouvernement fédéral dans la protection par l'État d'infrastructures civiles échappant à son autorité directe. Quelques mois plus tard, en mai 1998, dans le contexte des attentats contre les ambassades américaines à Nairobi et Dar Es Salaam, le président Clinton instaure par décret la politique de protection des infrastructures critiques, en reprenant les recommandations de la commission présidentielle ${ }^{84}$. Si le FBI voit ses compétences renforcées avec l'établissement en son sein du National Infrastructure Protection Center (NIPC), un rôle accru est dévolu aux agences civiles comme les départements de l'énergie ou du commerce. Ce dernier voit s'établir en son sein le Critical Infrastructure Assurance Office (CIAO), chargé de définir la mise en place de la politique de protection des infrastructures critiques. Établie en dehors de la sphère militaire, sans relever complètement des seules agences de sécurité intérieure, la protection des infrastructures critiques se construit à l'image des rapports de

82. Clinton W., Executive Order n¹3010: Critical Infrastructure Protection, Washington DC, The White House, 17 juillet 1996.

83. Les travaux de la commission présidentielle popularisent le terme de « cyberterrorisme » qui apparait au milieu des années quatre-vingt-dix et fait rapidement l'objet de travaux et d'analyses - généralement pessimistes, voire catastrophistes - de la part des think tanks américains, comme la RAND ou le CSIS. Voir Webster W., de Borchgrave A., Cybercrime, Cyberterrorism, Cyberwarfare: Averting an Electronic Waterloo, Washington DC, Center for Strategic and International Studies, 1998.

84. Clinton W., Presidential Directive Decision 63: Critical Infrastructure Protection, Washington DC, The White House, 22 mai 1998. 
force qui se sont joués au sein de la commission présidentielle pendant un an et demi et consacre la naissance d'une administration se voulant en réseau, dépourvue d'autorité centrale et composée de multiples agences, reflétant ainsi la dissémination des lieux de pouvoir au sein du gouvernement américain. $\mathrm{Ne}$ relevant ni complètement de la politique de défense, ni exclusivement des missions de police, la protection des infrastructures critiques évolue dans un secteur imprécis dépourvu d'autorité déterminée. Assumant le passage au nouveau paradigme de la gestion des risques dans la sécurité nationale, elle incarne un nouveau système de protection basé sur une étroite collaboration du secteur privé et des agences civiles afin que chacun réduise les vulnérabilités de ses propres systèmes.

\section{Le 11 septembre et la transformation sécuritaire}

La politique mise en place à la suite des attentats du 11 septembre 2001 constitue l'aboutissement de la dynamique de la bomeland security. La création en 2003 du Department for Homeland Security (DHS) entérine le bouleversement bureaucratique qu'elle sécrétait dès le milieu des années quatrevingt-dix, c'est-à-dire la désuétude du schéma d'organisation existant au cours de la Guerre froide. Déjà, la mise en place en octobre $2001 \mathrm{du}$ Council for Homeland Security et de l'Office for Homeland security s'inscrivait dans le droit fil des propositions issues, avant les attentats du 11 septembre, de parlementaires, d'experts ou de la Cour des comptes, tous demandant la création d'un centre de décision capable d'élaborer et de coordonner la stratégie à mettre en œuvre en matière de sécurité du territoire et de protection face à des menaces de type terroriste ${ }^{85}$. Dès la fin des années quatre-vingt-dix, l'ensemble des reproches adressés à l'organisation de la lutte contre le terrorisme sont compilés par la commission Hart-Rudman sur la sécurité nationale au XXI siècle ${ }^{86}$. Dans son dernier rapport rendu public en février 2001, cette commission préconise la création d'une National Homeland Security Agency (NHSA), avec pour responsabilité « la planification, la coordination et l'intégration des diverses activités du gouvernement américain impliquées dans la sécurité du territoire ${ }^{87}$. » La commission Hart-Rudman propose d'établir cette agence à partir des moyens déployés par la FEMA, redonnant à celle-ci les attributions de défense civile qu'elle possédait au cours de la Guerre froide. Les recommandations de la commission Hart-Rudman sont reprises dans la

85. Decker R., Combating Terrorism: Comments on Counterterrorism Leadership and National Strategy, Washington DC, General Accounting Office (GAO-01-556T), 27 mars 2001, pp. 14 ; Rabkin N., Combating Terrorism: Linking Threats to Strategies and Resources, Washington DC, General Accounting Office (GAO-T/NSIAD- 00-218), 26 juillet 2000, pp. 2-8 ; Rabkin N., Combating Terrorism: Issues in Managing Counterterrorist Programs, Washington DC, General Accounting Office (GAO-T/NSIAD- 00-145), 6 avril 2000, pp. 111.

86. United States Commission on National Security in the 21st Century (Hart-Rudman Commission), Road Map for National Security: Imperatives for Change (Phase III Report), Washington DC, 15 février 2001, p. x.

87. Ibid, p.15 
proposition de loi H.R. 1158 du 21 mars $2001{ }^{88}$. Deux autres propositions de lois, aux finalités similaires, sont déposées sur le bureau de la Chambre des représentants au début de l'année 2001 89. Dans la même veine, d'autres réformes touchant à la défense civile, comme la création du poste de conseiller présidentiel à la cybersécurité annoncée le 9 octobre 2001 ou la mise en place la semaine suivante du National Infrastructure Advisory Council (NIAC), justifiées toutes deux par la nécessité de répondre aux attentats du 11 septembre, ont été élaborées avant la chute des deux tours de New York ${ }^{90}$. Non seulement les réformes mises en place ont été élaborées avant le 11 septembre, mais l'organisation même du département de la Homeland Security souligne combien il repose sur les structures fondées sous l'administration Clinton. En opérant la fusion autour de la FEMA des structures dépendantes du National Infrastructure Protection Center (NIPC), du Critical Infrastructure Assurance Office (CIAO), ou de la FedCIRC, toutes créées dans la seconde partie des années quatre-vingt-dix, le DHS démontre qu'il n'est pas une structure ex nibilo issue des attentats du 11 septembre, mais plutôt la consécration institutionnelle d'un espace de sécurité apparu après la Guerre froide.

Du point de vue stratégique, la stratégie de la bomeland security de 2002 réaffirme la place centrale dévolue à la réduction de la vulnérabilité sur la prévention de la menace. Nonobstant la rhétorique sur la menace terroriste, il s'agit avant tout de défendre le pays contre l'ensemble des risques, étant entendu que «le besoin de protéger notre territoire n'est pas lié seulement à la menace terroriste actuelle, il est lié à notre vulnérabilité chronique 91 ». Mettant aussi l'accent sur les menaces de pandémies ou les catastrophes naturelles, la stratégie de la bomeland security consacre définitivement la gestion des risques dans l’approche de sécurité nationale. Les deux stratégies de 2003

88. 107ème congrès, H.R. 1158: To Establish The National Homeland Security Agency, Washington DC, House of Representatives, 21 mars 2001.

89. La proposition H.R. 525 du 8 février 2001 préconise la création d'un organisme de conseil directement relié au Président et chargé de la coordination de l'ensemble des mesures de préparation à la lutte contre le terrorisme. La proposition H.R. 1292 du 29 mars 2001 s'avère, quant à elle, à la fois plus large dans sa formulation mais moins ambitieuse en termes de réforme institutionnelle. Elle recommande en effet la désignation par le Président d'une personnalité au sein du gouvernement responsable de l'ensemble des questions relevant de la sécurité du territoire, c'est-à-dire de la protection contre une « attaque terroriste ou stratégique ». Pour une vision complémentaire sur ces trois propositions de loi, voir Decker R., Combating Terrorism: Observations on Options to Improve Federal Response, Washington DC, General Accounting Office, [GAO-01-660T], 24 avril 2001, p.1-7.

90. Le poste de conseiller du Président à la cybersécurité est accordé à l'ancien coordinateur national Richard Clarke. Les anciennes attributions de Richard Clarke concernant les missions traditionnelles du terrorisme sont transférées à un directeur national de l'anti-terrorisme (National Director for Combating Terrorism) dont le poste est également annoncé le 9 octobre 2001. Voir Rice C., Ridge T., "New Counter-Terrorism and CyberSpace Security Positions Announced”, Washington DC, The White House, Office of the Press Secretary, 9 octobre 2001. Sur le NIAC, voir Bush G.W., Executive Order n¹3231: Critical Infrastructure Protection in the Information Age, Washington DC, The White House, 16 octobre 2001, section 10, b) et c).

91. Bush G.W., The National Strategy for Homeland Security, Washington DC, The White House, Office of Homeland Security, juillet 2002, préambule. 
portant sur la protection des infrastructures critiques et la sécurité du cyberespace s'inscrivent dans la même perspective ${ }^{92}$. Si ces deux stratégies ont été présentées comme des réponses aux attentats du 11 septembre, leur élaboration était déjà prévue dès 1998. Il est d'ailleurs notable, pour aborder la dimension sociologique, que ces deux stratégies aient été écrites, non par des experts proches de l'administration Bush et nommés par elle, mais bien par les hommes qui avaient été installés par le président Clinton, au premier rang desquels Richard Clarke, ancien coordinateur national devenu conseiller du Président en matière de cybersécurité, et John Tritak, directeur du CIAO. A l'instar de ces hommes, c'est une nouvelle génération qui accède aux postes offerts par la création de la bomeland security, avec la promotion de personnalités comme Richard Falkenrath ou Franck Cillufo, deux exemples parmi d'autres d'experts ayant au cours des années quatre-vingt-dix expliqué en termes catastrophistes le danger du terrorisme de destruction de masse, pour le premier, ou du cyberterrorisme pour le second 93 .

\section{Conclusion}

La transformation contemporaine de la sécurité trouve ses origines dans des bouleversements plus profonds et plus anciens que les dramatiques événements du 11 septembre 2001. Une dynamique de sécurité s'est imposée sur les ruines de la Guerre froide en avançant une logique sécuritaire originale. En dépit des justifications officielles qui lui sont apportées, cette politique a peu à voir avec la violence politique telle qu'elle s'est exprimée avant et depuis le 11 septembre 2001. Si les attentats terroristes contribuent à lui assurer une forte légitimité, s'ils renforcent assurément ses dispositifs et accélèrent leur mise en place, la pérennité de cette politique n'est pas dépendante de l'occurrence des actes terroristes, car elle n'est pas structurée par une menace. Et il est probable qu'elle pourra s'adapter à tout nouveau danger qui ferait l'objet d'une attention particulière, comme elle l'a fait aux États-Unis avec les attentats à l'anthrax en 2001, encore non élucidés, ou avec les catastrophes naturelles après les dévastations du cyclone Katrina en 2005. Cette dynamique de sécurité, que nous avons tracée à partir du cas américain, mais dont les origines et les effets ne sauraient bien entendu se limiter à ce pays, influencent aujourd'hui forte-

92. The National Strategy to Secure Cyberspace, Washington DC, The White House, février 2003 ; The National Strategy for the Physical Protection of Critical Infrastructures and Key Assets, Washington DC, The White House, 2003.

93. Nommé dès janvier 2001 au poste de Director for Proliferation Strategy on the National Security Council Staff, Richard Falkenrath fut, à partir de 2003, Deputy Assistant to the President and Deputy Homeland Security Advisor. Franck Cilluffo fut nommé Special Assistant to the President for Homeland Security après les attentats du 11 septembre. Sur leurs écrits respectifs, voir Falkenrath R., Newman R., Thayer B., America's Achilles' Heel: Nuclear, Biological, and Chemical Terrorism and Covert Attack, Cambridge, Mass., MIT Press, 1998 ; Cilluffo F., "Information Terrorism and Strategic Terrorism", Terrorism and Political Violence, vol.9, n¹, 1997, pp. 84-94; de Borchgrave A., Cilluffo F., Cardash S., Ledgerwood M., Cyber Threats and Information Security: Meeting the 21st Century Challenge, Washington DC, Center for Strategic \& International Studies, 2001. 
ment les politiques de sécurité des pays occidentaux, et au-delà. Il reste toutefois comme perspective de recherche à déterminer deux facteurs. Premièrement, la capacité de cette dynamique de sécurité à demeurer pérenne. Si elle s'est renforcée à partir des liens entre les processus sociaux, ces derniers ne sont pas inexorablement amenés à converger. Les conditions qui ont permis de constituer le renouveau de la défense civile dans le contexte des années quatre-vingt-dix ne sont plus les mêmes aujourd'hui. Il convient alors de soupeser et d'analyser une décennie après les attentats du 11 septembre la manière dont évolue le champ de la sécurité à l'aune des transformations induites dès le milieu des années quatre-vingt-dix. Deuxièmement, il faudra qualifier davantage la nature de cette transformation. L'influence des experts du risque, le primat de la vulnérabilité sur la menace, les évolutions dans l'organisation même de l'État, les idées diffusées sur l'incertitude et le chaos, la généralisation des technologies de surveillance posent l'hypothèse de l'intégration de l'approche de précaution dans la sécurité nationale. 